\title{
Reminiscing on the origins of the field of progesterone and estrogen receptor action
}

\author{
Bert W O’Malley \\ Department of Molecular and Cellular Biology, Baylor College Medicine, Houston, Texas, USA
}

Correspondence should be addressed to B W O'Malley: berto@bcm.edu

This review forms part of a special section on 90 years of progesterone. The guest editors for this section are Dr Simak Ali, Imperial College London, UK and Dr Bert W O'Malley, Baylor College of Medicine, USA. Dr O'Malley was not involved in the peer-review process for the paper in which he is listed as an author.

\section{Memories of the discovery of steroid receptors}

The nuclear receptor (NR) field grew out of studies primarily of estrogen, progesterone, and glucocorticoid steroid hormones. The first indication for the potential existence of a steroid receptor occurred when Elwood Jensen injected radioactive estradiol into rats and noted a target tissuespecific uptake pattern which indicated the existence of an intracellular hormone-binding entity. This approach is detailed in a prior review (Jensen et al. 2010). This was followed by the demonstration that an estradiol-binding protein could be solubilized from uterus and characterized using a sucrose gradient by David Toft and Jack Gorski (Toft \& Gorski 1966). These two approaches solidified the existence of a macromolecular binding protein that was first named estraphilin due to skepticism among pharmacologists - and only later defined as a receptor. Estraphilin was shown to be located primarily in the nucleus during the target-tissue actions of the hormone. At this same time, we identified the progesterone receptor ( $\mathrm{PR}$ ) in oviduct and partially purified it and determined its biochemical and physical properties. The suspicion was that it was a mediator of some intracellular functions, likely the nuclear functions of progesterone. However, the molecular actions of progesterone and other steroids in the nuclei of eukaryotic cells remained unclear.

\section{Studies the mechanism of action of progesterone and estrogen receptors}

It was known from very early hormone injection experiments by Willard Myron Allen (Corner \& Allen, Am
J Physiol 1929; 88: 326-99), that progesterone could exert great influences on physiologic and pathologic functions of the ovary and uterus in mammals. It later became known that progesterone function is dependent upon a prior estrogen effect, and only later, that the cellular synthesis of progesterone receptor (PR) is regulated by estrogen. However, nothing was known of its mechanism of intracellular function. Consequently, and in concert with much later studies of the hormone binding by receptor proteins, there was great interest in discovering how hormones acted in cells. As clear as it appears to us in present time, understanding the 'mechanism of steroid action' was a slippery slope in those days. Competing theories in the 1960s were that steroids acted in the following ways: (1) as direct enzyme activators (Paul Talalay lab); (2) at the membrane to increase precursor/ nutrient uptake into cells (Oscar Hector lab); (3) posttranscriptionally to stabilize mRNAs (Gordon Tomkins lab); (4) on translation to increase protein synthesis (Tomkins lab); (5) via a second messenger such as cAMP(Earl Sutherland), or (6) on genetic DNA, similar to that proposed by Jacob and Monod for the lac operon in bacteria. Along with only a couple other labs, we strongly favored the latter hypothesis.

In 1967, our lab first published that progesterone induced de novo synthesis of the specific chick oviduct protein avidin; it did not just increase an enzyme activity but was proven to induce newly synthesized avidin protein in cell culture and in vivo. This was the 
first proven induction of synthesis of a specific target protein (not enzyme activity) by a steroid hormone. We next showed that steroid hormones stimulated RNA polymerase activity and the nuclear expression of 'new species' of RNA, findings that could not be explained by claims that hormones nonspecifically induced alterations in cytoplasmic compartments or in an increase in radioactive nucleotide precursor pools. By 1971, I published our data and my idea for the detailed the steps in the pathway of steroid hormone action; this summary remains essentially correct even today (O'Malley 1971). In a notable publication in 1972, we demonstrated that the synthesis of the specific mRNAs for ovalbumin (Means et al. 1972) and avidin could be induced by estrogen and progesterone, respectively. The following year, our work was confirmed and we went on to purify specific mRNAs and quantify their numerical concentrations by DNA-RNA hybridization technology. These discoveries substantiated the overall pathway for intracellular hormone action: that steroid hormones and receptors induce synthesis of specific proteins by first inducing synthesis of their specific mRNAs. Our mathematical calculations excluded the possibility of post-transcriptional mRNA regulation; translational regulation was excluded since there was no detectable pre-existing mRNA in oviduct polysomes in the absence of hormone stimulation. These studies resulted in the recruitment of many new investigators into the field and focused all future studies of hormone action and receptors and pharmaceutical development on the nucleus. This was the 'Birth of the Field of Molecular Endocrinology and Hormone Action' and steroid receptors and gene regulation became 'hot topics of study'. The later cloning of multiple steroid receptors by R. Evans (GR), P. Chambon (ER), O'Malley (PR) solidified the field and revealed a large number of additional related 'orphan receptors' (Evans) which eventually numbered 48 in humans, now termed as nuclear receptors (NRs).

When we purified the PR in the early 1970s, we surprisingly found that it has two isoforms, termed PRa and PRb. The PR receptor isoforms both bound to specific sequences in DNA which we later determined were activation sequences for nearby genes. The isoforms are dedicated to differing subsets of progesterone biology but cellular levels of both are dependent upon estrogen. After we cloned the PR and demonstrated its sequence and functional domains, we proceeded to develop an in vitro chromatin transcription system containing steroid target genes. Crude PR bound to DNA and appeared to have a stimulatory effect in this system, but when we used 'purified PR' in the absence of nuclear extract, the receptor was completely inactive. Considering that we had a great deal of evidence that PR was a transcriptional regulator in vivo, we were perplexed that the purified entity did not stimulate RNA synthesis in vitro. It appeared that some missing helper molecule(s) was present in the nuclear chromatin protein extract. However, we failed repeatedly in purifying this 'mystery factor'. The missing factor was the first indication for the existence of coactivators, and the reason we could not purify 'the' coactivator was because there were 'multiple' coactivators in the extract. In fact, it was representative of a giant family of coregulatory proteins that only later would be discovered.

\section{Discovery and cloning of coregulators for nuclear receptors}

The NR coregulators are composed of coactivators and corepressors. Coactivators are molecules which enhance transcription, and corepressors repress transcription. The first authentic nuclear receptor coactivator, SRC-1 (steroid receptor coactivator-1) (Onate et al. 1995), was discovered to be a type of transcription factor that did not bind DNA, but bound directly to PR to activate its transcriptional potential. We now believe that NRs are DNA-binding transcription factors that receive signals and simply locate target genes; in order for hormone 'action', the NRs must recruit coregulators to carry out all of the enzymatic reactions that are required to activate (or repress) genes. The importance of coregulators is paramount to the mechanism of NR action because they provide the biochemical functions needed to implement gene expression and the resultant cell physiologies.

The mechanisms of coregulators became more clear with a series of key proof-of-principle experiments. A mechanism by which NRs could repress transcription was first demonstrated when we introduced NRs into yeast cells; SSN6 bound to the activation domain of ER (or PR) and suppressed receptor transactivation; if this protein-protein interaction was prevented by mutations, the corepressor did not inactivate the receptor. NR activity was reactivated by hormone agonists but not by anti-hormones; thus, the hormones both promoted DNA binding of the NR and also induced a conformational alteration of the receptor that prevented the corepressor from functioning. Our first biochemical discovery of a soluble mammalian cellular 'corepressor' for a nuclear receptor (hTR) was published in 1995 and established the biochemical existence of this form of coregulator. The publication accurately predicted the now generally accepted concept for coregulator function: that 'corepressor exchange with a coactivator' 
is the mechanism for ligand-induced activation of nuclear receptors in primate cells. The existence of corepressors was substantiated in other labs by publications by the subsequent cloning of two specific and important general corepressors, NCoR and SMRT.

Yeast regulatory proteins also were valuable in proofof-principle experiments for coactivators. We found that a protein, termed SPT6 interacted specifically with the hormone binding domain of ER in vitro and in vivo and activated hormone induction of gene transcription. We identified several mammalian hormone-dependent interacting proteins for TR and ER, but none were purified or cloned and shown to have coactivator activity, until we cloned SRC-1 in 1994. Our studies with SRC-1 finally proved that 'recruitment of coactivators' was the missing step in the mechanism pathway for gene regulation by NRs. Our publications then established the still accepted criteria for coactivators: (1) coactivators bind to NRs; (2) agonist ligands induce coactivator binding to NRs; (3) antagonist ligands inhibit binding of coactivators to NRs; (4) coactivators create the full capacity of NRs to regulate transcription; (5) deletion of coactivator 'activation domains' (dominant negatives) inhibit endogenous ligand-dependent NR function in cells; (6) coactivators are the true physiologic regulators of NR actions in vivo in animals and have differing specific gene regulatory functions in cells; and finally, (7) coregulatory/corepressor ratios comprise the mechanism by which selective regulators of receptors (e.g., tamoxifen) act in a tissuespecific fashion.

An expanding number of publications of coactivators from many lab groups revealed coactivators to have diverse enzymatic functions. They also have been shown to regulate myriad non-receptor transcription factors. They function in multiprotein complexes that are recruited to a given gene in a NR-specific fashion. They contain the capacities required for chromatin remodeling, initiation and elongation of transcription, alternative RNA splicing, and even termination of transcription. Surprisingly, they also can control cellular reactions outside the nucleus such as mRNA translation, mitochondrial function and certain membrane growth factor receptor actions. Over the following decade, the number of coregulators that were cloned by numerous labs is now approaching >200 different molecules. This unveiling was accompanied by many studies that illustrated their importance as 'master genes' for the regulation of normal genetic physiology. As important physiological regulators, they also comprise important drivers for neoplastic pathologies when overexpressed.
Recently, we revealed the first structure (cryo-EM) of a full-length steroid receptor-SRC3-p300 complex on DNA - clearly a needed achievement for further clarification of NR and Coregulator biology. We then demonstrated structurally how 'coactivator exchange' within this complex occurs at the gene site so polymerase can move to subsequent stages of transcription such as elongation. Our lab then focused attention on coactivator-dependent disease mechanisms, demonstrating coactivator mediated cell motility, autophagy, metabolism, transformation and EMT, invasion and metastasis in cancers.

\section{Relationships of basic coactivator discoveries to endocrine physiology and disease}

Steroid receptors are the pharmacologic targets for $~ 15 \%$ of our current U.S.A. prescriptions. Our discovery that the cell coactivator/corepressor ratio determines 'tissuespecific' responses to SERM drugs was of importance in breast cancer therapy and highlighted coactivators as 'molecular markers' for cancer progression and therapy outcome. SRC-3 is the second most overexpressed oncogene among all human cancers, and miss-expressions of $>175$ coactivators have been associated with pathologies in 'patients', in cancers and other diseases.

In humans, the impressive recent associations of the 48 human NRs and the myriad coregulators influencing disease states marks them as one of the most important of all classes of intracellular regulators. Recent thoughts are to translate basic coregularor research into therapeutic benefit. In addition to cancer biology, it is clear that coregulator functions cut across all areas of medicine, including inherited diseases and genetic predispositions, metabolic regulation, reproduction, cardiovascular biology, neurobiology, aging, and toxicology. They comprise obvious markers for assessment of predisposition for diseases, as well as diagnosis and progression of diseases. Consequently, we believed that they could serve as great pharmaceutical targets for not only understanding current drug actions, but also for design of novel therapeutic interventions that could serve as future medical therapies.

Toward this end, we recently published that small molecule inhibitors (SMIs) can be discovered that directly target oncogenic SRC-3/SRC-1,2 coactivators; these SMIs selectively kill multiple types of cancer cells in vitro and in vivo. Separate discoveries currently are directed to SRCstimulators that markedly improve post-MI heart failure. Such discoveries serve as proof-of-principles to highlight a new 'first-in-class' type of drug target (against NR coactivators) not previously predicted. 


\section{Declaration of interest}

The author declares that there is no conflict of interest that could be perceived as prejudicing the impartiality of this commentary.

\section{Funding}

This article did not receive any specific grant from any funding agency in the public, commercial or not-for-profit sector.

\section{References}

Jensen EV, Jacobson HI, Walf AA \& Cheryl A 2010 Frye estrogen action: a historic perspective on the implications of considering alternative approaches. Physiology and Behavior 99 151-162. (https://doi. org/10.1016/j.physbeh.2009.08.013)

Means AR, Comstock JP, Rosenfeld GC \& O'Malley BW 1972 Ovalbumin messenger RNA of chick oviduct: partial characterization, estrogen dependence, and translation in vitro. PNAS 69 1146-1150. (https:// doi.org/10.1073/pnas.69.5.1146)

O'Malley BW 1971 Mechanisms of action of steroid hormones. New England Journal of Medicine 284 370-377. (https://doi.org/10.1056/ NEJM197102182840710)

Onate SA, Tsai SY, Tsai MJ \& O’Malley BW 1995 Sequence and characterization of a coactivator for the steroid hormone receptor superfamily. Science 270 1354-1357. (https://doi.org/10.1126/ science.270.5240.1354)

Toft D \& Gorski J 1966 A receptor molecule for estrogens: isolation from the rat uterus and preliminary characterization. PNAS 55 1574-1581. (https://doi.org/10.1073/pnas.55.6.1574)

Received in final form 13 February 2020

Accepted 16 March 2020

Accepted Manuscript published online 17 March 2020 (c) 2020 Society for Endocrinology Published by Bioscientifica Ltd. Printed in Great Britain 